\title{
Beneficial effect of "EldORost" on seed germination energy of wheat, mung beans and radish
}

Filippova O.I. ${ }^{1}$, Kulikova N.A. ${ }^{1}$, Konstantinov A.I. ${ }^{1}$, Grigoryeva I.O. ${ }^{1}$, Zhilkibayev O.T. ${ }^{2}$, Perminova I.V. ${ }^{1}$

${ }^{1}$ Lomonosov Moscow State University, Moscow, Russia

${ }^{2}$ Al-Farabi Kazakh National University, Almaty, Kazakhstan, zhilkibaevoral@mail.ru

doi: 10.36291/HIT.2019.filippova.061

"EldORost" is a universal organic fertilizer and natural plant growth regulator. It consist of salts of humic and fulvic acids (12\%), a complex of minerals and trace elements, amino acids, flavonoids and etc. Among the numerous hypotheses about the nature of the physiological activity of humic substances (HS), the hypothesis about their hormone-like action, which is primarily understood as an auxin-like effect, has recently become the most popular [1]. Therefore, HS is widely used in agriculture as a stimulator for plant growth at the early stages of their development. Our study was aimed to estimate effect of "EldORost" on germination energy of crops belonging three agricultural groups: grains (on the example of wheat Triticum aestivum L., cv. L1), legumes (on the example of mung bean Vigna radiata (L.) R.Wilczek. cv. "Pobeda-104") and small-seeded vegetables (on the example of radish Raphanus sativus L. var. sativus Cv. Krasnyi Velikan). To reach this goal, plant seeds were germinated in the presence of "EldORost" of different concentration; distilled water was used as a control.

The content of organic carbon (OC), humic and fulvic acids in "EldORost" measured using TOC analyzers Shimadzu (Japan) were 68, 59 and $9 \mathrm{~g}$ OC/l respectively. Meanweighted molecular mass was $7.4 \mathrm{kDa}$, and polydispersity was 3.4. Carbon distribution among structural fragments determined using 13C NMR spectroscopy demonstrated relatively high content of carboxylic groups (13\%) and aromatic structures (44\%). "EldORost" increased seed germination energy for all the studied plants (Figure 1).

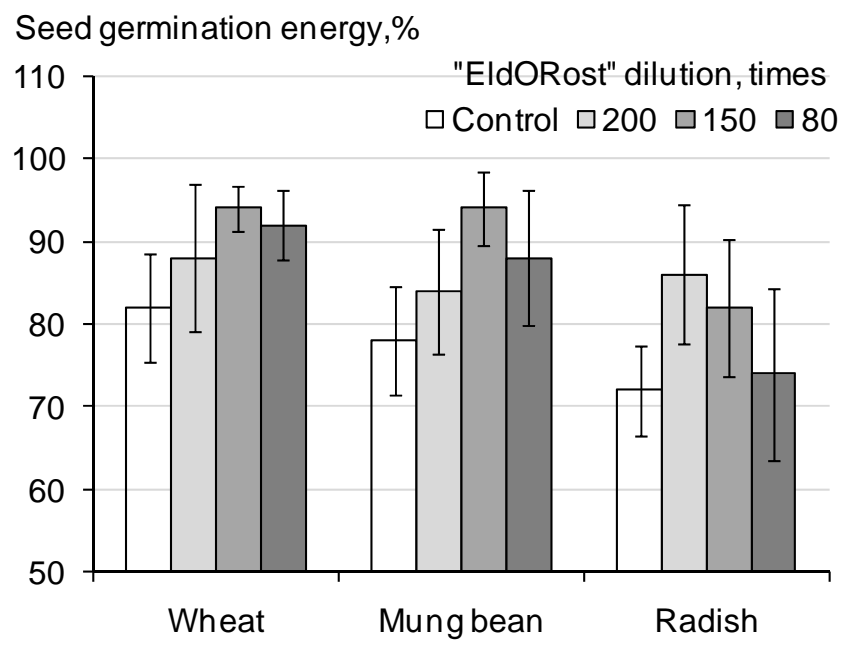

Figure 1. Effect of "EldORost" on germination energy.

Our results demonstrated that maximum beneficial effect of "EldORost" was observed when initial solution of plant growth stimulator was diluted 150 times, whereas more concentrated solution were less efficient. So, "EldORost" is a promising product for pre-sowing seed treatment.

\section{References}

1. Olaetxea M. et al. // Appl. Soil Ecol. 2018. 123:521-537. 